\title{
Haematological scoring system- a diagnostic tool for neonatal sepsis. How reliable is it?
}

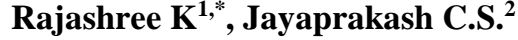 \\ ${ }^{\mathbf{1}}$ Assistant Professor, Dept. of Pathology, SS institute of Medical Sciences and Research Center, Davangere, Karnataka, \\ ${ }^{2}$ Professor, Dept. of Pathology, Father Muller Medical College, Mangalore, Karnataka, India
}

*Corresponding Author:

Email: drrajashree411@gmail.com

\begin{abstract}
Introduction: Neonatal septicaemia is the important cause for mortality and morbidity. Early diagnosis helps in timely intervention and favorable outcome. Although blood culture is gold standard, it has low sensitivity and delays the diagnosis. Hence formulation of rapid diagnostic tools that can indicate sepsis at earlier stage is important.

Objective: To evaluate performance of each parameter in the Hematological scoring system (HSS) in diagnosis of neonatal sepsis.

Materials and Methods: Blood samples from 130 clinically suspected neonates were subjected to sepsis screening tests. Differential count was performed on the peripheral smear to obtain immature cell count. Hematological scoring was performed. The sensitivity, specificity, positive predictive value (PPV) and negative predictive value (NPV) were calculated for each of the parameter with blood culture as Gold Standard.

Results: Out of 130 sepsis suspected neonates, 35.4\% showed culture positivity. Total polymorphonuclear count had highest sensitivity (89.6\%) and NPV (84.3\%) and immature to mature PMN count (I:M) $>0.3$ had highest specificity (100\%)and PPV(100\%). HSS with the combination of two test being positive (score $\geq 2$ ) gave good sensitivity (100\%) and NPV (100\%); but low specificity (54.7\%) and PPV (54.7\%). Specificity and PPV increased with increase in the HSS.

Conclusion: No parameters can be taken alone as indicator of sepsis. Total PMN count was the sensitive indicator but lacks specificity. HSS score of <2 indicated unlikely sepsis, 2-4 probable sepsis, $\geq 5$ most likely sepsis. HSS can be derived from single test is faster, easily available in most of the centers and hence allows early decisions regarding antibiotic therapy.
\end{abstract}

Keywords: Neonatal sepsis, Haematological scoring system, Blood culture, Immature polymorphonuclear cells.

\section{Introduction}

Infections are a frequent and important cause of neonatal morbidity and mortality. ${ }^{1}$ Neonatal sepsis is a clinical syndrome of bacteremia characterized by systemic signs and symptoms of infection in the first month of life. It encompasses systemic infections of the newborn including septicemia, meningitis, pneumonia, arthritis, osteomyelitis and urinary tract infection of the newborn. ${ }^{2}$ The earliest signs of sepsis are often subtle and nonspecific and need a high index of suspicion for early diagnosis. Neonatal sepsis is diagnosed when generalized systemic infection is associated with growth of bacteria from one or the other sites. ${ }^{3}$

According to WHO estimates, Neonatal Sepsis is the commonest cause of neonatal mortality and is probably responsible for $30-50 \%$ of the total neonatal deaths each year in developing countries. It is estimated that $20 \%$ of all neonates develop sepsis and approximately $1 \%$ die of sepsis related complications. ${ }^{4}$ Clinical diagnosis of neonatal sepsis may be difficult as the early signs of the sepsis may be subtle and different. Although blood culture is considered gold standard diagnostic tool, it takes about $48-72 \mathrm{hrs}$ for the report, resulting in delay in treatment. Hence it is important to formulate the diagnostic modalities which help in early diagnosis of sepsis in neonates.

Although many recent studies show diagnostic tests (such as procalcitonin, fibronectin, interleukin 6) with high sensitivity, but they are impractical because of high cost and sophisticated methods.

Hematological scoring system includes various hematological parameters derived from single test (compete hemogram), which show changes during the sepsis. It is simple, quick, cost effective and readily available tool in early diagnosis of neonatal sepsis. The study aims at evaluating the performance of different parameters in hematological scoring system in diagnosis of neonatal sepsis.

\section{Materials and Methods}

This prospective study was conducted in the Department of Pathology, Father Muller Medical College Hospital, Mangalore from October 2011 to December 2012. Blood samples were collected from clinically suspected cases of neonatal septicemia admitted to the Neonatal Intensive Care Unit, Father Muller Medical College Hospital, constituted the material of the study. Detailed history and clinical findings were recorded. Study included all the neonates with clinical suspicion of sepsis based on clinical findings and prenatal risk factors. Neonates born to known immunocompromised mother, suspicion of torch, malarial infections, with congenital abnormities, who received antibiotics before drawing blood for culture were excluded from the study.

The blood samples were collected in EDTA Vaccutainer tubes. Peripheral blood smears were be 
prepared, stained with Leishman stain and examined under oil immersion light miceroscopy at a final magnification of X1000. The sepsis work up included blood culture, routine blood counts along with the hematologic score. Total leucocyte count was obtained using Beckman Coulter and corrected for nucleated red blood cells. Differential counts including the immaute polymorphonulear (PMN) leucocytes were performed on Leishman stained smear.
The Hematological scoring system (HSS) (table 1) designed by Rodwell et $\mathrm{al}^{5}$ was used for obtaining a score in each case. A score of one was assigned for each abnormal value of seven parameters found to be significantly associated with sepsis with one exception. An abnormal total count is assigned a score of 2 instead of 1 , if no mature polymorphs are seen on the peripheral smear to compensate for the low I:M ratio.

Table 1: Hematological scoring system

\begin{tabular}{|l|c|c|c|}
\hline S. No & Criteria & Abnormality & Score \\
\hline 1 & Total WBC count & $<5000 / \mu 1$ & 1 \\
& & $>25,000$ at birth & 1 \\
& & $>30,000$ at $12-48 \mathrm{hr}$ & 1 \\
\hline 2 & Total PMN ratio & $1800-5400 / \mu 1$ & 0 \\
& & No mature PMN seen & 2 \\
& Increased/decreased & 1 \\
\hline 3 & Immature PMN count & $<600 / \mu 1$ & 0 \\
& & Increased & 1 \\
\hline 4 & Immature: Total PMN ratio & $<0.12$ & 0 \\
& (I:T) & $>0.12$ & 0 \\
\hline 5 & Immature: Mature PMN & $<0.3$ & 1 \\
\hline 6 & ratio (I:M) & $>0.3$ & 1 \\
\hline 7 & Degenerative changes in & Toxic granules/ Cytoplasmic & 1 \\
\hline
\end{tabular}

Blood culture was done under aseptic precautions using bactec technology. Subculture was done on subsequent days. It was considered negative if no organism were detected upto 5 days of inoculation.

Blood culture was taken as gold standard for diagnosis of sepsis. Parameters results were compared with the blood culture to determine number of True positives (TP), False positives (FP), True negatives (TN) and False negatives (FN) cases. Sensitivity, specificity, positive predictive accuracy and Negative predictive accuracy were calculated using the above values. Data analysis were performed using the SPSS software for Windows (Statistical System Software, SPSS Inc, 1999, New York) version 10.0.

\section{Results}

The present study enrolled 130 cases of neonates with clinical suspicion of sepsis. Among 130 cases, 46 were positive for blood culture studies with success rate of $35.4 \%$.

Out of 46 culture positive cases, $26(56.5 \%)$ were male and $20(43.5 \%)$ were females. Male to female ratio was $1.3: 1$. Majority $(n=38,82.6 \%)$ of the neonates were less than 1 week. The common risk factors associated with culture positive cases were low birth weight $(\mathrm{n}=21,50 \%)$ and gestational age $<37$ weeks $(\mathrm{n}=16,34.7 \%)$

On evaluating performance of each haematological parameter in haematological scoring system (Table 2), none of the parameter had acceptable sensitivity and specificity together. Total polymorphonuclear count had highest sensitivity and negative predivtive value $>80 \%$. The Immature to total polymorphonuclear count, immature to mature ratio and degenerative changes in neutrophils had specificity and positive predictive value of $>95 \%$ and $>80 \%$ respectively.

Table 3: Performance of each hematological parameters of hematological scoring system in diagnosis of neonatal sepsis

\begin{tabular}{|l|c|c|c|c|}
\hline Tests & $\begin{array}{c}\text { Sensitivity } \\
(\boldsymbol{\%})\end{array}$ & $\begin{array}{c}\text { Specificity } \\
(\boldsymbol{\%})\end{array}$ & $\begin{array}{c}\text { Positive } \\
\text { predictive } \\
\text { value }(\boldsymbol{\%})\end{array}$ & $\begin{array}{c}\text { Negative predictive } \\
(\boldsymbol{\%}) \text { value }\end{array}$ \\
\hline Total Wbc count & 47.8 & 83.3 & 61.1 & 74.4 \\
\hline Total PMN count & 89.1 & 32.1 & 41.8 & 84.3 \\
\hline Immature PMN count & 67.3 & 63 & 50 & 77.9 \\
\hline I:T PMN ratio & 30.4 & 96.4 & 82.4 & 71.6 \\
\hline
\end{tabular}




\begin{tabular}{|l|c|c|c|c|}
\hline I:M PMN ratio & 13 & 100 & 100 & 67.7 \\
\hline $\begin{array}{l}\text { Degenerative changes in } \\
\text { PMN }\end{array}$ & 17.3 & 97.6 & 80 & 68.3 \\
\hline Platelet count & 36.9 & 88 & 62.9 & 71.8 \\
\hline
\end{tabular}

On combining the different parameters and obtaining a score, 9 culture positive cases showed the score of $0-1$. Where as 24 culture positive cases had score of 3 and 4. Six culture positive cases showed score $>5$. As the score increased, specificity and positive predictive value increased (Fig. 1).

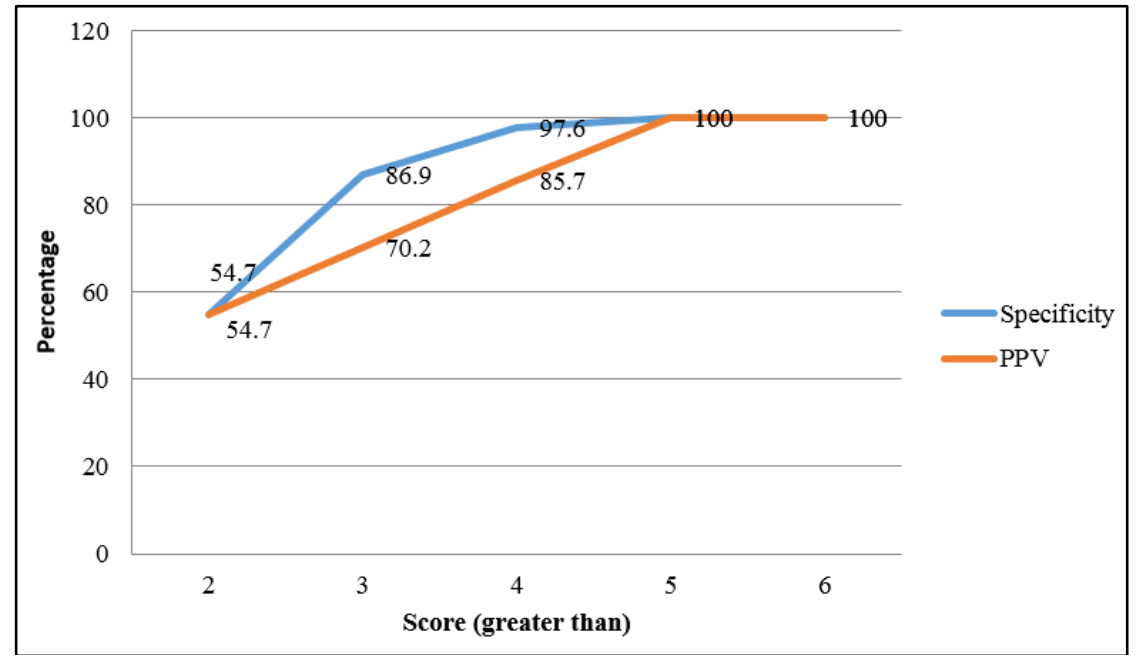

Fig. 1: Performance of hematological scoring system

\section{Discussion}

Neonatal septicemia continues to be a major cause of morbidity and mortality in our country. It is one of the major causes of neonatal mortality in the developing countries contributing to $15 \%$ of all neonatal deaths. Although, a life-threatening condition, yet it can treatable in ease if diagnosed early. Unfortunately, the early warning signs and symptoms are often nonspecific and can easily be confused with those from noninfective causes. These nonspecific signs and symptoms make it difficult to establish an early clinical diagnosis.

In recent years, various investigators have evaluated some highly sensitive and specific inflammatory markers (eg. ELIZA methods, haptoglobins, interleukins and counterimmunoelectrophoresis etc,) to diagnoseneonatal sepsis. Although, these markers are sensitive and specific, but are sophisticated and expensive so impractical for developing countries. Therefore, the need is for a test that is cheap easily performed with quick availability of reports.

The present study showed male predominance with $\mathrm{M}: \mathrm{F}$ ratio of $1.3: 1$, which was in concordance with studies done by Vinodkumar C.S.et al, ${ }^{6}$ Shah et al. ${ }^{7}$ It is attributed to the fact that, there are $\mathrm{x}$ linked regulatory genes involved in immune regulation, males have single $\mathrm{X}$ chromosome, making them more susceptible for infections..
Neonates at first week of life are more susceptible to infections because of their immature immunological response to infections ${ }^{1}$. Early onset neonatal sepsis was more common compared to late onset neonatal sepsis which was also observed by Vinodkumar C.S. et al, ${ }^{6}$ Tallur et al. ${ }^{8}$

Total leucocyte count had high sensitivity but lacked specificity, where as total polymorphonuclear count had specificity and negative predictive value but lacked sensitivity. These findings were similar to study done by Khair et al. ${ }^{9}$ They found that Total leukocyte count (TLC) is of little clinical use in the diagnosis of neonatal infection because of wide variation in values. TLC may not show any changes in cases due to early collection, laboratory errors in counting.

According to Xantou, ${ }^{10}$ In babies with undoubted and very severe infective illness, along with the rise in the neutrophils there was nearly always a significant rise in the more immature forms sometimes exceeding 1000/cumm. They also reported that immature cell count dropped significantly before death. Similar to observation made by Makkar et $\mathrm{al}^{11}$ Immature polymorphonuclear count had low specificity but high sensitivity.

Immature to total count ratio had specificity and positive predictive value of $100 \%$ similar to studies done by Misquith et al. ${ }^{12}$ Many other studies have shown high sensitivity in this parameter., ${ }^{9,14}$ The differences may be due to diagnostic criteria followed, interobserver variation in counting immature cells, time of sample collection. 
Ghosh et $\mathrm{al}^{13}$ showed that the degenerative changes in neutrophils had high specificity and low sensitivity as observed in our study.

Neonatal thrombocytopenia is a common clinical problem in nicu various factors lead to low platelet count in neonates. In a study done by Sonam et $\mathrm{al}^{15}$ the causes for thrombocytopenia were prematurity, sepsis, respiratory distress syndrome, birth asphyxia, hyperbilirubenemia, and intrauterine growth retardation. In our study thrombocytopenia had low sensitivity but high positive predictive value and specificity similar to observations made by Narashimha et al. ${ }^{14}$

In our study, although Score of 1 and 2 had sensitivity of $100 \%$ but lacked high specificity, where as score more than 4 had good specificity and positive predictive value. As the score increased the specificity and positive predictive value increased indicating likelihood of sepsis at higher scores. Various others studies showed similar results. ${ }^{9.14}$

\section{Conclusion}

Hematological scoring system is easy, faster, one step test, cost effective tool for diagnosing haematological sepsis. None of the parameters can be used alone as predictive marker. Combining the scores and obtaining the score might help in early diagnosis and initiation of the treatment. A HSS score of 0-2 indicated unlikely sepsis, 3-4 indicated probable sepsis where as score of 5 and 6 indicate sure sepsis.

\section{References}

1. Barbara J Stoll. Infections of the neonatal infant. In: Nelson textbook of paediatrics. 17th ed. Philadelphia: WB Saunders Company;2004.794-809

2. Aggarwal R, Sarkar N, Deorari AK, Paul VK. Sepsis in the Newborn. Indian J Pediatr. 2001;68(12):1143-47.

3. Meherban Singh. Care of the newborn. 5th ed. New Delhi: Sagar publications;1998.769-804.

4. WHO. Perinatal mortality. Report No.: WHO/FRH/MSM/967. Geneva: WHO, 1996.

5. Rodwell RL, Leslie AL, Tudehope DI. Early diagnosis of neonatal sepsis using a hematologic scoring system. $J$ Pediatr. 1988;112:761-67.
6. Vinodkumar CS, Neelagund YF, Kalsurmath S, Banapurmath S, Kalappannavar NK, Basavarajappa KG. Perinatal risk factors ad microbial profile of neonatal septicemia: A multicentred study. J Obstet Gynecol India. 2008;58(1):32-40.

7. Shah GS, Budhathoki S, Das BK, Mandal RN. Risk factors in Early neonatal sepsis. Katmandu University Medical Journal. 2006;4(2):187-91.

8. Tallur S, Kasturi AV, Shoba D, Krishna BVS. Clinicobacteriological Study of neonatal septicemia in Hubli. Indian J Pediatr. 2000;67(3):169-74.

9. Khair KB, Rahman MA, Sultana T, Roy CK, Rahman MQ, Ahmed AN. Early diagnosis of neonatal septicemia by hematologic scoring system, C-reactive protein and serum haptoglobin. Mymensingh Med J. 2012;21(1):8592.

10. Xanthou M. L eucocyte blood picture in healthy full term and premature babies during neonatal period. 1970. Arch Dis Child. 45:242-49.

11. Makkar M, Guptha C, Pathak R, Garg S, Mahajan N.C. Performance evaluation of Hematological scoring system in early diagnosis of neonatal sepsis. J Clin Neonatology. 2011.2(1):25-9.

12. Ronald Misquith, Prakash Saldanha, Varadaraj Shenoy K, Sanjeev Rai B. The Use of Buffy Coat Smear for Diagnosis of Neonatal Septicaemia. Karnataka Pediatric Journal. 2004;18(1):9-13.

13. Ghosh S, Mittal M, Jagaanathan G.Early diagnosis of neonatal sepsis using a Hematological scoring system. Ind J Med Sci. 2001;55(9):495-500.

14. Narasimha A, Harendra Kumar ML. Significance of Hematological Scoring System (HSS) in Early Diagnosis of Neonatal Sepsis. Indian J Hematol Blood Transfus. 2011;27(1):14-17.

15. Nandyal $S^{2}$, P. Shashikala, Sahgal V. Study of thrombocytopenia in neonatal intensive care unit. Indian $J$ Path and Oncology. 2016;3(1):55-9.

How to cite this article: Rajashree $\mathrm{K}$, Jayaprakash CS. Haematological scoring system- a diagnostic tool for neonatal sepsis. How reliable is it?. J Diagn Pathol Oncol. 2018;4(3):219-222. 